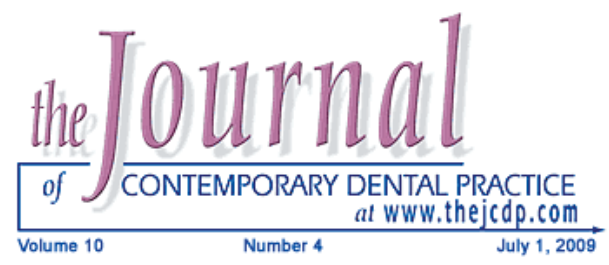

\title{
Palatal and Labially Impacted Maxillary Canine-associated Dental Anomalies: A Comparative Study
}

\section{Archna Nagpal, MDS; Keerthilatha M. Pai, MDS;} Gaurav Sharma, MDS

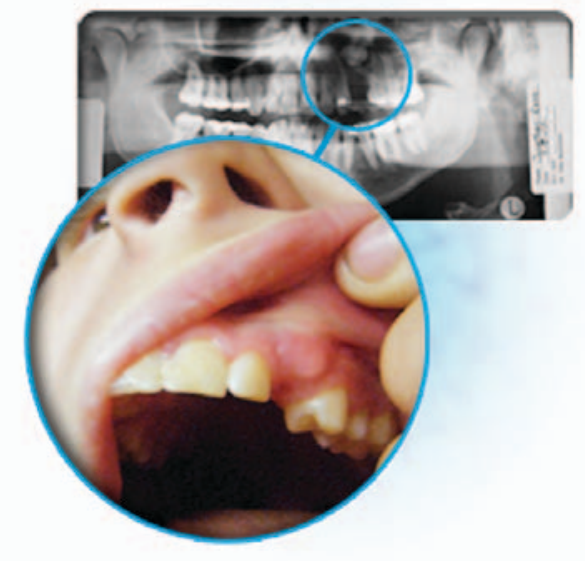

\section{Abstract}

Aim: To determine the association of impacted maxillary canines with other developmental dental anomalies and compare them with a control population and to compare palatally and labially impacted canine associated anomalies.

Methods and Materials: Ninety non-syndromic orthodontic patients from a south Indian population, with either labial or palatal impactions of one or both maxillary canine teeth, were selected for the study group. A group of 250 patients who had normally erupted maxillary canines were selected as controls. Clinical findings and panoramic radiographs were used to identify dental anomalies.

Results: Prevalence of premolar rotation was found to be $80 \%$ in the study group followed by canine rotation $(20 \%)$, taurodontism $(26.6 \%)$, infraocclusion of premolars $(25.5 \%)$, impacted mandibular canine $(11.1 \%)$, and peg lateral $(7.7 \%)$. Forty-nine (54.4\%) palatally impacted, $37(41.1 \%)$ labially impacted, and $4(4.4 \%)$ centrally impacted canines were also observed. Of the 250 subjects in the control group $78.8 \%$ presented with premolar rotation. Maxillary canine transmigration, infraocclusion of premolars, anomalies of maxillary lateral incisors, and taurodontism showed significant associations with palatally impacted canines $(P<0.05)$.

Conclusion: The present study illustrates a significant association of anomalous maxillary lateral, anomalous mandibular canine, taurodontism, infra occlusion of premolar, over retained deciduous molar, and maxillary

(c) Seer Publishing 
canine transmigration with ectopically placed canines. These associations suggest these conditions may share a common genetic origin and existence of these anomalies can anticipate maxillary canine impaction which can help in cautious observation and early treatment for maxillary canine impaction.

Clinical Significance: Anomalous lateral incisors can serve as a predictor for palatal maxillary canine impaction. These anomalies could serve as an easily recognized, early warning sign for the monitoring of the canine position and timely early referral in appropriate cases.

Keywords: Impaction, palatal, labial, anomalies

Citation: Nagpal A, Pai KM, Sharma G. Palatal and Labially Impacted Maxillary Canine-associated Dental Anomalies: A Comparative Study. J Contemp Dent Pract 2009 July; (10)4:067-074.

\section{Introduction}

No tooth is more interesting from a developmental point of view than a maxillary canine. Of all teeth, it has the longest period of development, the deepest area of development in alveolar bone, and the most devious course to travel from its point of origin to full eruption into occlusion. The maxillary canine tooth in man is sometimes disturbed from its usual position within the dentoalveolar process, disrupting its normal eruption, often resulting in non-emergence. Most canine impactions diverge from the normal eruptive site in either a palatal or labial direction.

The etiology of maxillary canine impaction has been ascribed to many different causes with one being local to the canine area. ${ }^{1,2}$ Miller $^{3}$ and Bass $^{4}$ suggested a local disturbance of the dental lamina might be responsible for setting the canine

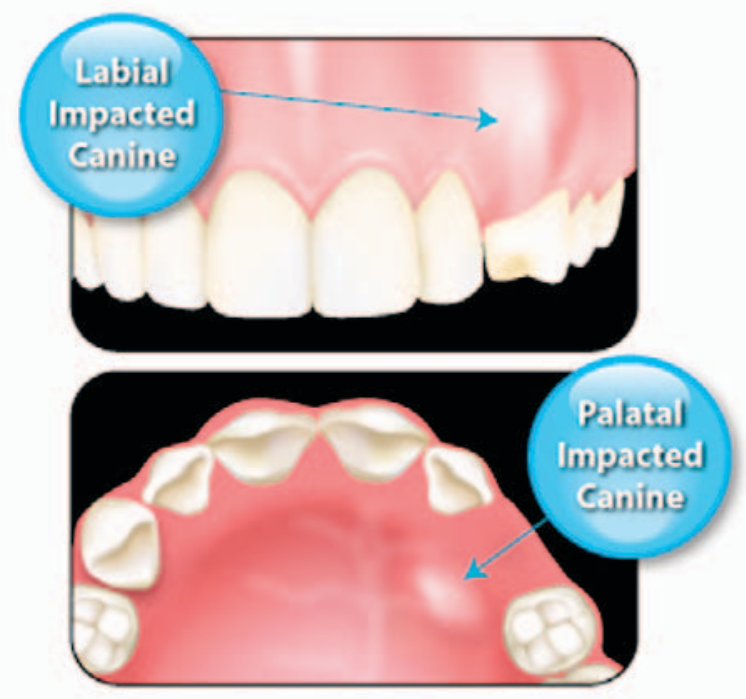

on the wrong eruption pathway. Takahama and Aijjama suggested canine impaction might be a manifestation of a microform of cleft lip and palate. ${ }^{5}$

The cause and effect associations most often suggest palatal canine impaction occurs as a result of a number of local conditions and factors as follows:

1. An over-retained deciduous canine

2. Anomalous and missing lateral incisors

3. A crowded or delayed eruptive pathway

However, a palatally impacted canine seems to occur in most cases with normal form and occlusion of the teeth. Jacoby ${ }^{6}$ concluded a canine could be palatally displaced if extra space is available in the maxillary bone. Such space can be provided by the following mechanisms:

1. Excessive growth in the base of the maxillary bone

2. Space created by agenesis or peg-shaped lateral incisors

3. Stimulated eruption of the lateral incisor or first premolar

In the classical description of the permanent maxillary canine eruption path, the root of the permanent lateral incisor plays a cardinal role. However, when the erupting canine fails to obtain a normal position it is often impacted. This may be due to unsatisfactory guidance of the lateral incisor root, excessive space in the maxilla, or for any other reason.

Becker et al. and Brin et al. have constructed arguments implicating lateral incisor variations 
in the genesis of the palatal development of permanent maxillary canines. ${ }^{7,9}$ They claim peg shaped, short rooted, or absent maxillary lateral incisors are causal factors in the development of palatally impacted canines. In contrast, this could not be substantiated by Brenchly ${ }^{10}$ with statistically significant evidence to support the view palatally impacted maxillary canines are associated with diminutive maxillary lateral incisors.

Genetic factors may be involved in the etiology of tooth eruption disturbances. Zilberman et al. ${ }^{11}$ concluded from their study in 1990 family members of patients with palatal canines are likely to exhibit palatally impacted canines, anomalous lateral incisors, absence of crowding, and late developing dentitions. Bjerklin and coworkers investigated the associations among four anomalies (ectopic eruption of first molars, infraocclusion of deciduous molars, ectopic eruption of maxillary canines, aplasia of premolars) in $1992 .^{12}$ They found ectopic eruption of maxillary canines had a significantly higher prevalence than expected in all the other three groups. They interpreted the four conditions studied to be different manifestations of one syndrome with each manifestation having an incomplete penetrance.

According to Peck et al. ${ }^{13}$ in 1994 the palatally impacted canine appeared to be a developmental anomaly with genetic determinants. Then in 1996 the same investigators stated the anomalies of tooth agenesis, tooth size reduction, and palatally impacted canines are biologic covariables in a complex of genetically related dental disturbances. ${ }^{14}$

The results of the study by Baccetti ${ }^{15}$ corroborate the genetic hypothesis of the etiology of the anomalous position of maxillary canine. Palatal impacted canines showed significant reciprocal associations with small-size maxillary lateral incisors, aplasia of the second premolar, infraocclusion of deciduous molars, and enamel hypoplasia.

In 1989 a study by Olivier, et al. ${ }^{6}$ concluded maxillary lateral incisors adjacent to an impacted canine is generally smaller than the contralateral incisor. This occurred in a Caucasian population with predominantly palatal canine impactions and in an Oriental population with predominantly labial impactions. Svinhufvud et al. ${ }^{17}$ suggest an autosomal dominant inheritance pattern for ectopic eruption (including palatal and labial impactions) of the maxillary canine since it is closely related to hypodontia.

Evidence has been accumulating to suggest biology links some dental abnormalities occurring together more frequently than would be expected by chance alone. These related anomalies include variations in tooth number, size, and eruption chronology and sequencing. All these are identified as manifestations of some shared genetic mechanisms.

The etiology of impacted teeth has been a controversial subject. The two canine malpositions, labial and palatal impactions, are actually very different phenomena although they seldom have received separate considerations in studies of impacted canines. Labial displacement is usually due to inadequate arch space and it eventually results in eruption in most cases. In contrast, a palatally impacted maxillary canine is a positional anomaly generally occurring despite sufficient arch space and characteristically results in an impaction of the tooth.

Evidence has been mounting regarding the genetic association of palatally impacted canines, but labially impacted canines have received limited attention as a covariable in the pattern of association among dental anomalies. Hence, the present investigation was undertaken to determine the association of impacted maxillary canine (palatal or labial) with other developmental dental anomalies and compare them with a control population. Furthermore, a comparison was made between palatally and labially impacted canine associated anomalies and to indicate the etiologic and clinical relevance of such associations.

\section{Methods and Materials}

The material for this study was obtained from records of several orthodontic facilities in the cities of Manipal and Mangalore, Karnataka, India. These included both branches of the Manipal College of Dental Sciences in Manipal and in Mangalore; the Yenepoya Dental College in Mangalore; the AB Shetty Institute of Memorial 
Sciences in Mangalore; and two private orthodontic offices in Mangalore. The study group comprised of 90 nonsyndromic orthodontic patients from a south Indian population with either labial or palatal impactions of one or both maxillary canine teeth. The tube-shift technique was used in all the cases to determine the location of palatal or labial maxillary canine impactions. A group of 250 patients (age, gender, and race matched to the study group), whose maxillary canines had erupted normally, were selected as controls. Clinical examination and panoramic radiographs were used to identify developmental dental anomalies.

\section{Inclusion Criteria}

The inclusion criteria for subjects in the study and control groups consisted of the following:

- Panoramic radiographs of good quality

- Clinical records

- Use of the tube-shift technique for localization of impactions

\section{Exclusion Criteria}

The exclusion criteria consisted of the following:

- Complex craniofacial malformations or syndromes

- Cleft lip and palate

- Sequelae of traumatic injuries to the teeth

- Multiple or advanced caries

- Racial diversity

- Familial relationships with other examined subjects (twins and siblings)

- Hormonal disturbances such as hypothyroidism and hypopituitarism

The distribution of type of malocclusion was Class I (52.7\%), Class II (26.6\%), and Class III (14.4\%) in the study group and Class I (63.6\%), Class II (28.8\%), and Class III (7.2\%) in the control group. The type of malocclusion could not be assessed for one subject each in the study and control groups since the permanent mandibular molar was impacted in those cases.

The prevalence rates of dental anomalies were noted in relation to the impacted canines in both the study and control groups. The following dental anomalies were evaluated:

1. Congenitally missing lateral incisors or other congenitally missing teeth.

2. Supernumerary tooth and odontome.

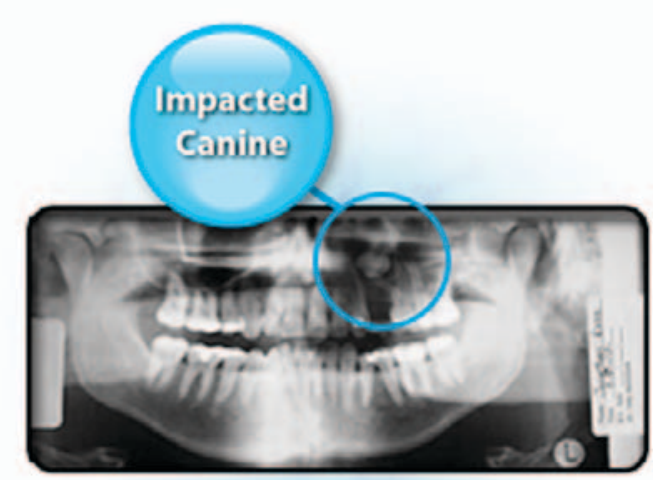

3. Taurodontism. This was evaluated by measuring the crown-body length (distance from the central pit on the occlusal surface to the furcation) as well as the root length (furcation to the root tip) of the mandibular first permanent molar using a panoramic radiograph. The diagnosis was confirmed if the crown-body to the root length ratio was greater than 1:1 in accordance with the recommendation of Seow and Lai. ${ }^{18}$

4. Over retained deciduous teeth.

5. Transposition. An interchange in the position of two adjacent teeth within the same quadrant of the dental arch. In an incomplete transposition, the crowns may be transposed while the root apices remain in their normal positions.

6. Small permanent lateral incisor. Defined as small, mesiodistally, if its shape was normal but smaller than its mandibular counterpart.

7. Peg lateral. A peg shaped lateral was recorded when mesiodistal dimensions of lateral incisors were greatest at the cervical margin.

8. Tooth rotations

9. Infraocclusion. The diagnosis was confirmed when the occlusal surface of the affected tooth was located below the occlusal plane.

10. Transmigration. A pre-eruptive migration of a tooth across the midline. In the maxilla it was recorded if the crown of a canine had crossed the mid-saggital plane when confirmed using an occlusal radiograph.

Intra-examiner assessment was also done after one week of examination. The $Z$ test of proportionality was used to statistically compare 
the prevalence of a dental anomaly in the study group with the control group and the prevalence of dental anomalies in palatally and labially impacted canines.

Level of significance was considered as follows:

- $Z \geq 1.96, p<0.05$

- $Z \geq 2.58, p<0.01$

- $Z \geq 3.29, p<0.001$

The Chi-square test was performed to detect the association of anomalies of maxillary lateral incisors, impacted teeth, missing teeth, and supernumerary teeth with labially and palatally impacted canines.

\section{Results}

At the time of detection of the maxillary impacted canine, the age range was 13 to 25 years with a mean age of 14.3 years. There were right sided (31), left sided (28), and bilateral (31) maxillary canine impactions. Forty-nine $(54.4 \%)$ palatally impacted, 37 (41.1\%) labially impacted, and 4 $(4.4 \%)$ centrally impacted canines were observed (Table 1).

A re-examination of the records of all 90 subjects was performed one week following the initial examination to assess the reproducibility of the diagnosis. Reproducibility was complete for all dental anomalies except for the following:

- Premolar rotation (96.67\%)

- Small maxillary lateral incisor $(96.67 \%)$

- Infraocclusion of premolars $(94.56 \%)$

- Taurodontism (93.23\%)

Forty-four (17.6\%) subjects had no radiographic evidence of dental abnormality in the control group. Table 2 compares the percentages of dental anomalies in the study group to the findings in the control population in the same geographic area.

When the control group was compared with the study group, maxillary transmigration, small maxillary lateral incisor, taurodontism, and mandibular canine rotation all demonstrated highly significant associations with maxillary canine impactions $(p<0.001)$. At the same time, peg laterals and over-retained deciduous mandibular canines had statistically significant associations with impacted maxillary canines $(p<0.01)$. Anomalies such as over-retained deciduous molars, impacted lateral and congenitally missing lateral incisors also had a significant association with ectopically placed maxillary canines $(p<0.05)$.

Four patients had central canine impaction. Table 3 compares the prevalence rates of dental anomalies in palatally and labially impacted canines. Maxillary canine transmigration $(p<0.01)$, infraocclusion of premolars $(p<0.05)$, and taurodontism $(p<0.05)$ all showed significant associations with palatally impacted canines. When different categories of anomalies of lateral incisors (Microdont, peg lateral, impacted lateral, rotated lateral, congenitally missing lateral) among labially and palatally impacted canines were compared, a statistically significant association $(p<.05)$ was found with palatally impacted canines. No other significant associations were found when a comparison was made with palatally and labially impacted maxillary canine associated anomalies.

Although $80 \%$ of subjects in the study group presented with premolar rotations, this was not associated with impacted maxillary canines since $78.8 \%$ of the control group subjects also had premolar rotations.

Table 1. Distribution of maxillary impacted canines.

\begin{tabular}{|c|c|c|c|}
\hline \multicolumn{2}{|c|}{$\begin{array}{c}\text { Study Group } \\
\text { (n=90) }\end{array}$} & \multicolumn{2}{c|}{$\begin{array}{c}\text { Study Group } \\
(n=90)\end{array}$} \\
\hline $\begin{array}{c}\text { Left Sided } \\
(28)\end{array}$ & $\begin{array}{c}\text { Right Sided } \\
(31)\end{array}$ & $\begin{array}{c}\text { Palatal } \\
(49)\end{array}$ & $\begin{array}{c}\text { Labial } \\
(37)\end{array}$ \\
\hline \multicolumn{2}{|c|}{$\begin{array}{c}\text { Bilateral } \\
(+31)\end{array}$} & \multicolumn{2}{|c|}{$\begin{array}{c}\text { Central } \\
(4)\end{array}$} \\
\hline
\end{tabular}


Table 2. Table compares the percentages of dental anomalies in study group to the findings in the control population in the same geographic area.

\begin{tabular}{|c|c|c|c|c|c|}
\hline & \multicolumn{2}{|c|}{$\begin{array}{c}\text { Control Group } \\
\text { (250) }\end{array}$} & \multicolumn{2}{|c|}{$\begin{array}{l}\text { Study Group } \\
(90)\end{array}$} & \multirow[t]{2}{*}{$\begin{array}{c}\text { Level of } \\
\text { Significance }\end{array}$} \\
\hline & & $\%$ & & $\%$ & \\
\hline Premolar rotation & 197 & 78.80 & 72 & 80 & NSS \\
\hline Mandibular canine rotation & 10 & 4 & 18 & 20 & $p<0.001$ \\
\hline Maxillary lateral incisor rotation & 0 & 0 & 3 & 3.33 & NSS \\
\hline Maxillary central incisor rotation & 0 & 0 & 1 & 1.11 & NSS \\
\hline Transmigration (maxillary) & 0 & 0 & 9 & 10 & $p<0.001$ \\
\hline Transmigration (mandibular) & 1 & 0.40 & 3 & 3.33 & NSS \\
\hline Supernumerary tooth & 3 & 1.20 & 1 & 1.11 & NSS \\
\hline Odontome & 3 & 1.20 & 3 & 3.33 & NSS \\
\hline Small maxillary lateral incisor & 4 & 1.60 & 18 & 20 & $p<0.001$ \\
\hline Peg lateral incisor & 1 & 0.40 & 7 & 7.77 & $P<0.01$ \\
\hline Displaced maxillary lateral incisor & 4 & 1.60 & 18 & 20 & NSS \\
\hline Congenitally missing maxillary lateral incisor & 0 & 0 & 4 & 4.44 & $P<0.05$ \\
\hline Impacted maxillary lateral incisor & 0 & 0 & 4 & 4.44 & $P<0.05$ \\
\hline Infra-occlusion (premolars) & 6 & 2.40 & 23 & 25.55 & NSS \\
\hline Infraocclusion (mandibular canine) & 5 & 2 & 4 & 4.44 & NSS \\
\hline Microdontia & 10 & 4 & 1 & 1.11 & NSS \\
\hline Over-retained deciduous lateral & 0 & 0 & 2 & 2.22 & NSS \\
\hline Over-retained deciduous molar & 3 & 1.20 & 6 & 6.66 & $P<0.05$ \\
\hline Over-retained deciduous mandibular canine & 0 & 0 & 5 & 5.55 & $P<0.01$ \\
\hline Impacted permanent molar & 1 & 0.40 & 1 & 1.11 & NSS \\
\hline Impacted premolar & 1 & 0.40 & 7 & 7.77 & NSS \\
\hline Impacted mandibular canine & 1 & 0.40 & 10 & 11.11 & NSS \\
\hline Impacted maxillary lateral incisor & 0 & 0 & 1 & 1.11 & NSS \\
\hline Taurodontism & 10 & 4 & 24 & 26.66 & $p<0.001$ \\
\hline Transposition & 0 & 0 & 2 & 2.22 & NSS \\
\hline Congenitally missing premolar & 0 & 0 & 2 & 2.22 & NSS \\
\hline Congenitally missing mandibular lateral incisor & 0 & 0 & 1 & 1.11 & NSS \\
\hline
\end{tabular}


Table 3. Compares the prevalence rates of dental anomalies in palatally and labially impacted canines.

\begin{tabular}{|c|c|c|c|c|c|}
\hline & \multicolumn{2}{|c|}{$\begin{array}{l}\text { Palatal Subjects } \\
\text { (49) }\end{array}$} & \multicolumn{2}{|c|}{$\begin{array}{l}\text { Labial Subjects } \\
\text { (37) }\end{array}$} & \multirow[t]{2}{*}{$\begin{array}{c}\text { Level of } \\
\text { Significance }\end{array}$} \\
\hline & & $\%$ & & $\%$ & \\
\hline Small maxillary lateral incisor & 13 & 26.53 & 5 & 13.51 & NSS \\
\hline Peg lateral incisor & 5 & 10.20 & 1 & 2.70 & NSS \\
\hline Maxillary lateral displaced incisor & 3 & 6.12 & 1 & 2.70 & NSS \\
\hline Congenitally missing maxillary lateral incisor & 3 & 6.12 & 1 & 2.70 & NSS \\
\hline Impacted maxillary lateral incisor & 1 & 2.04 & 0 & 0 & NSS \\
\hline Rotated premolars & 38 & 75.55 & 31 & 83.78 & NSS \\
\hline Rotated mandibular canine & 10 & 22.44 & 7 & 18.91 & NSS \\
\hline Rotated maxillary central incisor & 1 & 2.04 & 0 & 0 & NSS \\
\hline Rotated maxillary lateral incisor & 3 & 6.12 & 0 & 0 & NSS \\
\hline Transposition & 1 & 2.04 & 1 & 2.70 & NSS \\
\hline Maxillary canine transmigration & 7 & 14.28 & 0 & 0 & $p<0.01$ \\
\hline Mandibular canine transmigration & 1 & 2.04 & 2 & 5.40 & NSS \\
\hline Taurodontism & 9 & 16.32 & 14 & 37.83 & $p<0.05$ \\
\hline Infraocclusion of premolars & 9 & 16.32 & 14 & 37.83 & $p<0.05$ \\
\hline infraocclusion of mandibular canine & 3 & 6.12 & 1 & 2.70 & NSS \\
\hline Supernumerary tooth & 1 & 2.04 & 0 & 0 & NSS \\
\hline Odontome & 0 & 0 & 3 & 8.10 & NSS \\
\hline Spacing & 30 & 61.22 & 26 & 70.27 & NSS \\
\hline Impacted mandibular canine & 4 & 8.16 & 6 & 16.21 & NSS \\
\hline Impacted molar & 1 & 2.04 & 0 & 0 & NSS \\
\hline Missing maxillary canine & 3 & 6.12 & 0 & 0 & NSS \\
\hline Missing maxillary premolar & 2 & 4.08 & 0 & 0 & NSS \\
\hline Missing mandibular central incisor & 1 & 2.04 & 0 & 0 & NSS \\
\hline Impacted premolar & 1 & 2.04 & 5 & 13.51 & NSS \\
\hline
\end{tabular}


Discussion

The present study focused on impacted maxillary canines and coexisting dental anomalies.

A correlation was found between impacted maxillary canine and the dental anomalies of tooth number, tooth size, over retained deciduous teeth, rotations, and infraocclusion. This study confirms the findings of the earlier workers regarding the role of anomalous lateral incisors in maxillary canine impactions. ${ }^{7,8}$ Various theories and hypothesis have been offered regarding the reasons for palatal maxillary canine impaction. These include both local and genetic factors. Little has been reported in the literature regarding the causation of labially impacted canines, and a review of the dental literature indicates no study has been done to compare anomalies associated with labially and palatally impacted canines.

The role of the root of the lateral incisor is to serve as a guide for the normal eruption of the maxillary cuspid; this is being pointed out to emphasize Broadbent's original observation in 1941. ${ }^{19}$ Miller suggested even a rudimentary lateral incisor can develop a root of sufficient length to provide the guidance needed for normal eruption of the cuspid. ${ }^{3}$ However, according to Garn et al. ${ }^{20}$ teeth with a small mesiodistal dimension usually develop late which means a lateral incisor will be insufficiently developed to provide critical guidance during the very early stage of development and migration of the cuspid. However, once the slowly forming lateral incisor has developed it can block the "corrective" buccal migration of the cuspid. Any rotation of the lateral incisor would, therefore, guide the canine labially or palatally, depending on the direction of rotation, but it is impossible to state if this is a cause or an effect of the canine impaction. Even though these arguments all emphasize the local factor involved in impaction, the clinician should be mindful that abnormalities of the lateral incisors are strongly under genetic control.

Anomalies of maxillary lateral incisors, aplasia, or impaction of other teeth and the presence of a cross-bite may serve as indicators of palatal canine displacement. ${ }^{21}$ An earlier study stressed the role of an anomalous maxillary lateral incisor in a maxillary canine palatal impaction. ${ }^{8}$ Bacetti $^{14}$ revealed a significant reciprocal association between a small size maxillary lateral incisor and maxillary impacted canine in a study conducted in 1998.

In the present study, 28 of 49 palatally impacted canines demonstrated an anomalous adjacent lateral incisor in contrast to only eight of 37 labially impacted canines. The latter could have been due to mechanical obstruction of the erupting canines.

The findings of the study pointed out a significant correlation between mandibular canine rotation $(p<0.001)$ over retained deciduous mandibular canine $(p<0.01)$ and impacted maxillary canines. In the study group, ten samples showed occurrence of mandibular canine impaction (unilateral or bilateral), concomitantly with maxillary canine impaction (not statistically significant). There is no plausible explanation for this phenomenon, but heredity can be suggested as a possible cause. The developmental anomaly of taurodontism showed a statistically significant $(p<0.001)$ association with impacted maxillary canines in this study. Lai and Seow ${ }^{18}$ found significant associations of hypodontia with ankylosis of deciduous molars, taurodontium, enamel hypoplasia, and peg-shaped incisors in 1989. It is not surprising to find a significant association with an impacted canine, since it has a close relation with peg laterals.

The significant association with overretained deciduous molar and infraocclusion of premolars point out the lateness of development of dentition with an impacted canine. This attribute confirms the findings of previous authors. ${ }^{11}$ Studies by others ${ }^{12}$ considered the infraocclusion of deciduous teeth as one covariable but fail to explain a deciduous tooth will always be in infra occlusion with the complete eruption of adjacent permanent teeth.

Most of the anomalies in the present study failed to depict any significant associations when palatally and labially impacted canine groups were compared. Only maxillary canine transmigration, infraocclusion of premolars, taurodontism, and anomalous lateral incisor showed significant associations with palatal canine impaction (Table 3). No other significant associations were found when a comparison was made with palatally and 
labially impacted maxillary canine associated anomalies.

The clinical impression was palatally impacted canines seemed to have an affinity for the spaced dentition, ${ }^{11}$ but this could not be confirmed in the present study. This could be due to the lack of defined criteria for spacing in the arch in the study.

Maxillary canine transmigration was found to be significantly associated with palatally impacted canines. The reason for this finding could be the impacted tooth follows a path of least resistance represented by the excessive space available palatally into which the canine can easily migrate. The roots of the maxillary incisors are very prominent labially which function as an obstruction to the normal eruption pathway.

Impaction of various teeth (lateral incisors, mandibular canines, molars, and premolars) were found in association with labially impacted canines. The reason for such occurrence is unclear.

One shortcoming of the present study is the lack of segregation of the subjects on the basis of type of malocclusion because the incidence of canine impaction varies among different occlusion types.

\section{Conclusion}

The present study illustrates significant associations between ectopically placed canines and anomalous maxillary laterals, mandibular canines, taurodontism, infraocclusion of (rotated, overretained) premolars, and over retained deciduous molars. These associations suggest these conditions may share a common genetic origin, and existence of these anomalies can anticipate maxillary canine impaction which can help in cautious observation and early treatment for maxillary canine impaction.

\section{Clinical Significance}

As with many other inherited anatomic conditions, early recognition and interception are valuable strategies in conservative clinical management of impacted maxillary canines. Anomalous lateral incisors can serve as a predictor for palatal maxillary canine impaction. These anomalies could serve as an easily recognized, early warning sign for the monitoring of the canine position and timely early referral in appropriate cases. 


\section{References}

1. Rayne J. The unerupted maxillary canine. Dent Pract Dent Rec 1969; 19 (6);194-204.

2. Kay LW. The impacted maxillary canine. Dent Update. 1977 Sep; 4(6):335-9.

3. Miller BH. Influence of congenitally missing teeth on eruption of the maxillary canine. Transactions of the British society for the study of the orthodontics 1963; 50;17-24.

4. Bass TB. Observations on the misplaced canine tooth. Dental Practitioner 1967; 18;25-33.

5. Takahama Y, Aiyama Y. Maxillary canine impaction as a possible microform of cleft lip and palate. Eur J Orthod 1982 Nov; 4(4):275-7.

6. Jacoby $\mathrm{H}$. The etiology of maxillary canine impactions. Am J Orthod 1983; 84;125-132.

7. Becker $A$, Smith $P$, Behar R. The incidence of anomalous lateral incisors in relation to palatally impacted cuspids. Angle Orthod 1981; 51;24-29.

8. Becker A, Zilberman Y, Tsur B. Root length of lateral incisors adjacent to palatally impacted maxillary cuspids. Angle Orthod 1984; 54;218-225.

9. Brin L, Becker A, Shalhav M. Position of the maxillary permanent canine in relation to anomalous or missing lateral incisors: a population study. Eur J Orthod 1986; 8;12-16.

10. Brenchly Z, Oliver G. Morphology of anterior teeth associated with impacted canines. Br J Orthod 1997; 24;41-5.

11. Zilberman $\mathrm{Y}$, Cohen B, Becker A. Familial trends in palatal canines, anomalous lateral incisors, and related phenomena. Eur J Orthod. 1990 May; 12(2):135-9.

12. Bjerklin $\mathrm{K}$, Kurol J, Valentin J. Ectopic eruption of maxillary first permanent molars and association with other tooth and developmental disturbances. Eur J Orthod. 1992 Oct; 14(5):369-75.

13. Peck S, Peck L, Kataja M. The palatally impacted canine as a dental anomaly of genetic origin. Angle Orthod 1994; 64(4):249-56.

14. Peck S, Peck L, Kataja M. Site-specificity of tooth agenesis in subjects with maxillary canine malpositions. Angle Orthod. 1996; 66(6):473-6.

15. Bacetti T. A controlled study of associated dental anomalies. Angle Orthod 1998 Jun; 68(3):267-74.

16. Oliver RG, Mannion JE, Robinson JM. Morphology of the maxillary lateral incisor in cases of unilateral impaction of the maxillary canine. Br J Orthod 1989 Feb; 16(1):9-16.

17. Svinhufvud E, Myllärniemi S, Noria R. Dominant inheritance of tooth malpositions and their association to hypodontia. Clin Genetics 1988; 34;373-381.

18. Lai PY, Seow WK. A controlled study of the association of various dental anomalies with hypodontia of permanent teeth. Pediatr Dent. 1989 Dec; 11(4):291-6.

19. Broadbent BH. Ontogenic development of occlusion. Angle Orthod 1941; 11;223.

20. Garn S, Lewis AB, Vicinus JH. Effect of agenesis on the crown-size profile pattern. Dent Res 1963; 24;1344-1363.

21. Leifert S, Jonas IE. Dental anomalies as a microsymptom of palatal canine displacement. J Orofac Orthop. 2003 Mar;64(2):108-20.

\section{About the Authors}

\section{Archna Nagpal, MDS}

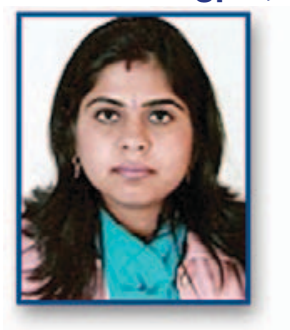

Dr. Nagpal is a Senior Lecturer in the Department of Oral Medicine and Radiology of the Sri Gobind Tricentenary Dental College in Gurgaon, India. Her research interests include dental anomalies and digital radiology.

e-mail: drarchnanagpal@ rediffmail.com 
Keerthilatha M. Pai, MDS

Dr. Pai is a Professor and Head, Department of Oral Medicine and Radiology, Manipal College of Dental Sciences, Manipal, Karnataka, India. Her research interest is in oral pre-cancer detection.

e-mail: keerthilatha.pai@manipal.edu

Gaurav Sharma, MDS

Dr. Sharma is a Senior Lecturer in the Department of Oral Medicine and Radiology, ITS Dental College, Ghaziabad, UP, India.

e-mail: drgaurav7479@ rediffmail.com 